\title{
Thoracomyoplasty and perioperative negative wound pressure therapy for bronchopleural fistula after trans-sternal bronchial closure
}

\author{
Jinwook Hwang ${ }^{1,2}$, Jeong In Hong' ${ }^{1}$ Hong Ju Shin' ${ }^{1}$ Jae Seung Shin', Je Hyeong Kim ${ }^{2,3}$
}

Departments of ${ }^{1}$ Thoracic and Cardiovascular Surgery and ${ }^{2}$ Critical Care Medicine, and ${ }^{3}$ Division of Pulmonology, Department of Internal Medicine, Korea University Ansan Hospital, Korea University College of Medicine, Ansan, Korea
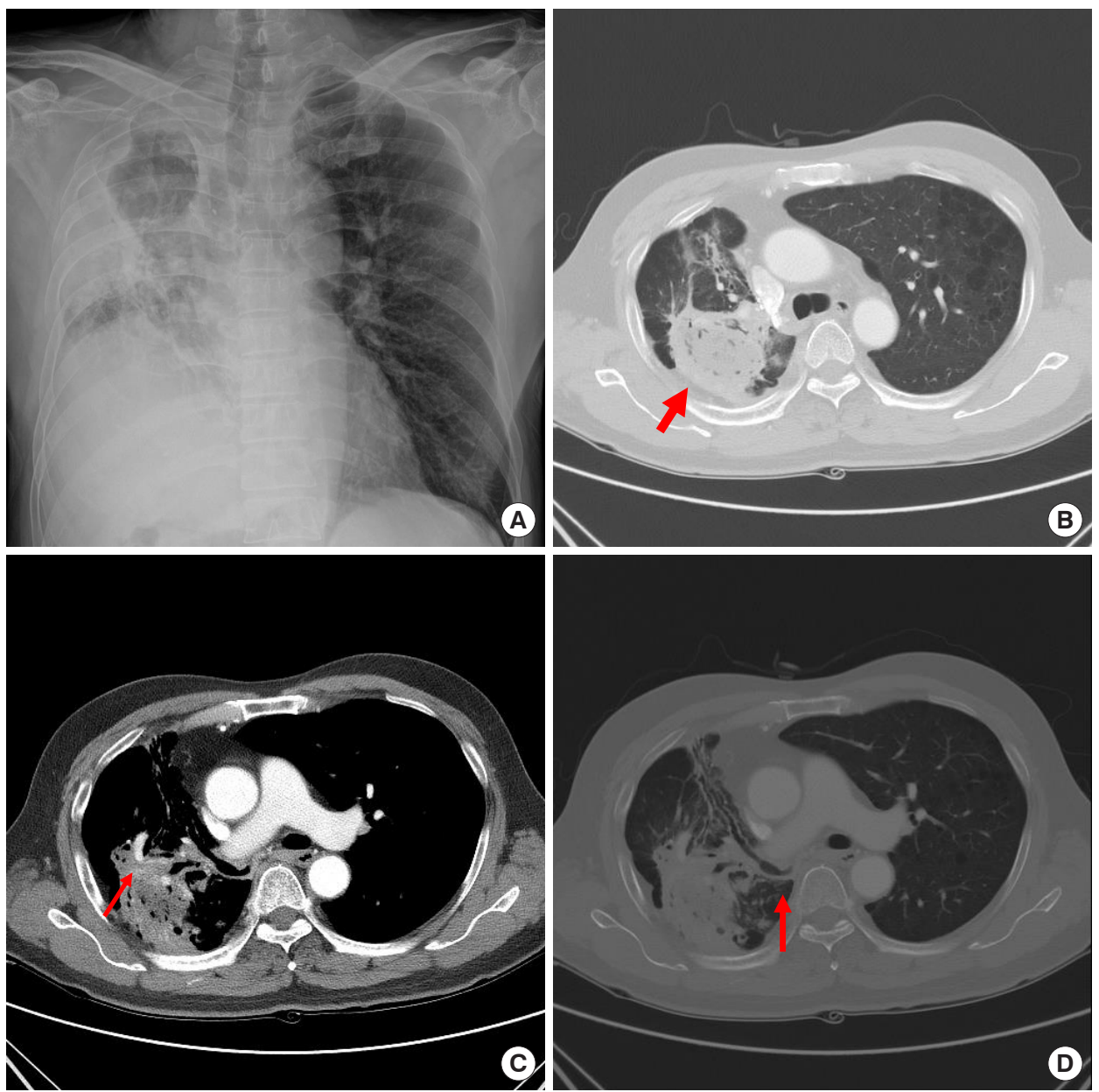

Figure 1. The chest images showed $(A, B)$ the right-side fungal ball (red arrow) and $(C)$ aneurysmal change of the bronchial artery (red arrow). (D) The right main pulmonary artery was divided with surgical material and only the right-side lower lobe bronchus was intact (red arrow).

\section{Image in Critical Care}

Received: May 19, 2021

Revised: May 24, 2021

Accepted: May 25, 2021

\section{Corresponding author} Je Hyeong Kim

Department of Critical Care Medicine, Korea University Ansan Hospital, Korea University College of Medicine and Division of Pulmonology, Department of Internal Medicine, Korea University Ansan Hospital, 123 Jeokgeum-ro, Danwon-gu, Ansan 15355, Korea Tel: +82-31-412-5950

Fax: +82-31-413-5950

E-mail: chepraxis@korea.ac.kr

Copyright () 2021 The Korean Society of Critical Care Medicine

This is an Open Access article distributed under the terms of Creative Attributions Non-Commercial License (https://

creativecommons.org/li-censes/by-nc/4.0/) which permits unrestricted noncommercial use, distribution, and reproduction in any medium, provided the original work is properly cited. 

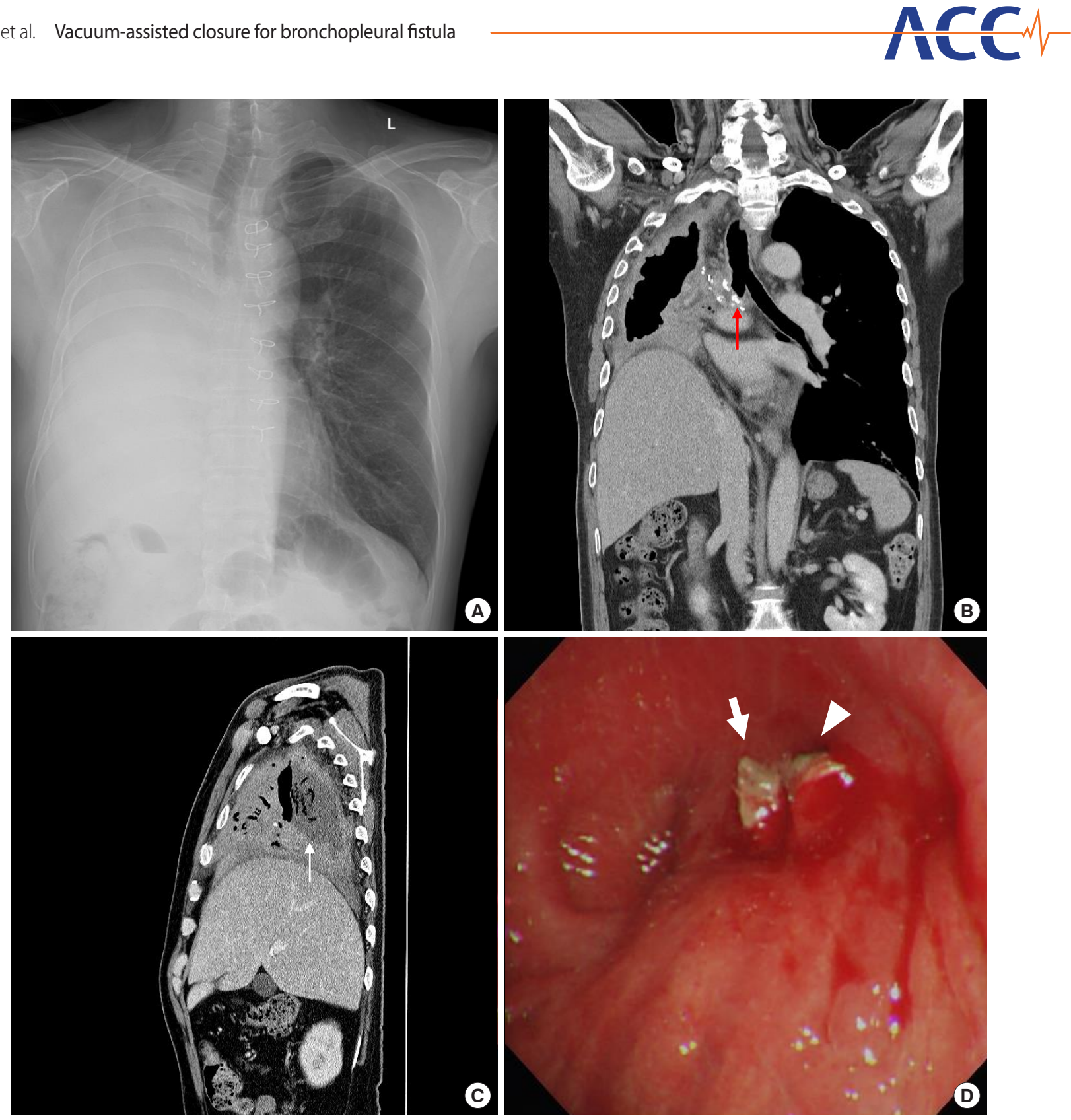

Figure 2. Two months after the trans-sternal bronchial division operation, the patient complained of a massive amount of purulent sputum. (A, B) The chest images showed a long right-side bronchial stump (red arrow) and cavitary change of the right-side thoracic cavity. (C) The fungal ball (white arrow) was still inside. (D) Bronchoscopy showed surgical material at the right-side bronchial stump (white arrow) and opening (arrowhead).

A 62-year-old male complained of recurrent hemoptysis from a right-side pulmonary fungal ball (Figure 1). His right main pulmonary artery was accidentally sacrificed during a bilobectomy 10 years ago, and the remnant lung had a cavitary lesion. We performed trans-sternal transpericardial bronchial closure to avoid pneumonectomy through the infected thoracic cavity and to control the patient's bloody cough [1]. The bronchial stump was closed using a surgical stapler and reinforced with Prolene sutures with felt pledgets. There were no postoperative complications, and the patient was discharged on postoperative day 13. Two months later, a bronchopleural fistula developed at the bronchial stump (Figure 2). The patient had undergone video-assisted thoracosopic surgery irrigation and complete pneumonectomy (Figure 3) [2-4], and then dead spaces were obliterated via a thoracomyoplasty procedure and perioperative negative pressure wound therapy (Figure 4) [5]. The last outpatient follow-up was 1 year later. The wound remained clean, and the patient's performance level was not impaired relative to his preoperative state. In the absence of standardized protocols for managing such pa- 

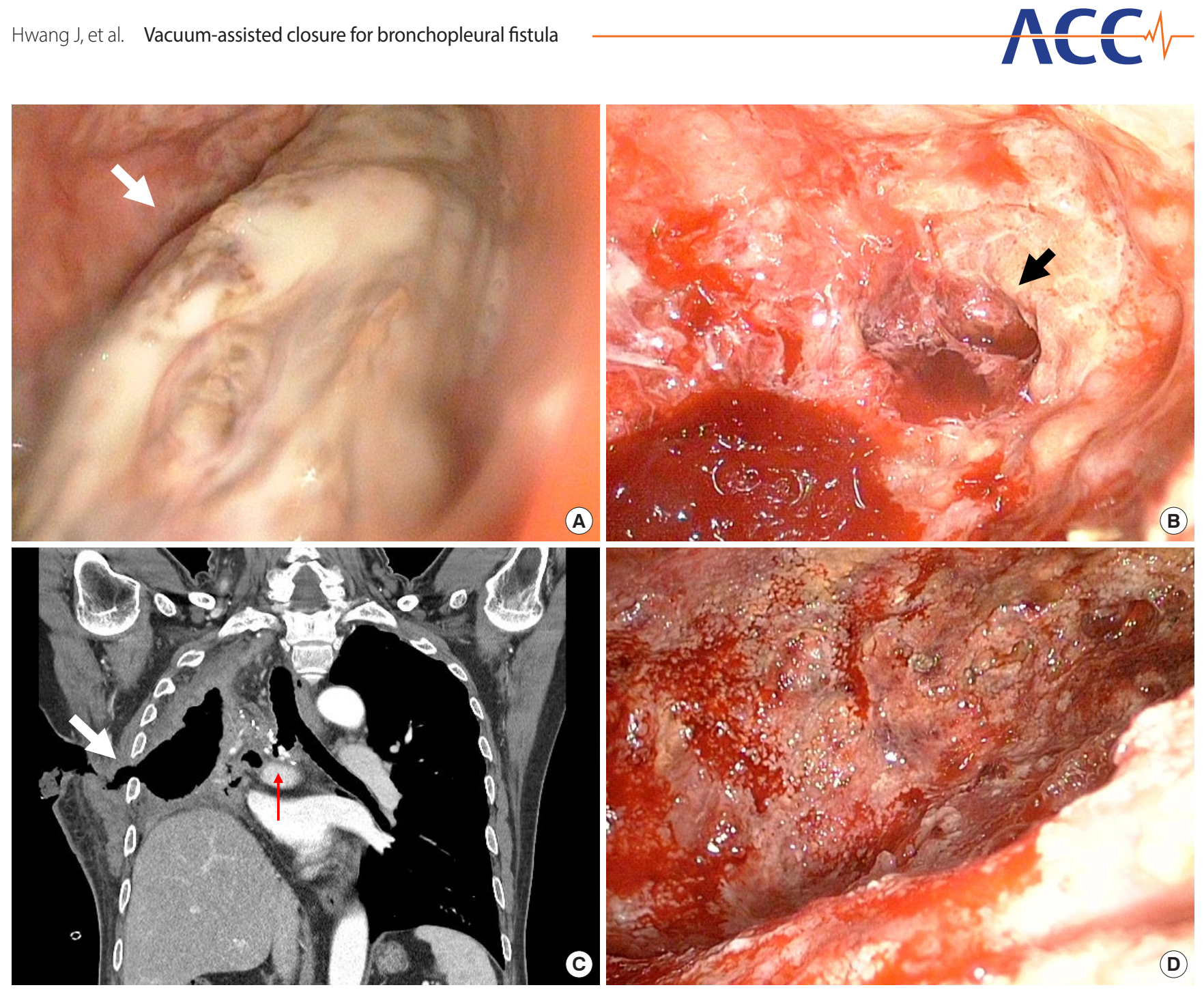

Figure 3. Video-assisted thoracoscopic exploration showed the fungal ball (white arrow) inside the right pulmonary cavity (A) and necrosis of the lung parenchyma (black arrow) after saline irrigation (B). (C) Chest computed tomography showed a bronchopleural fistula (red arrow) after trans-sternal trans-pericardial bronchial division and closure and an open wound (white arrow) of the chest wall after irrigation and drainage. (D) After exchanging a vacuum-assisted closure sponge under local anesthesia every 2 or 3 days, we performed follow-up pleural fluid culture every 3 days and found no bacterial growth.

tients, our reported case offers a review of these classic procedures that hybridize a minimally invasive technique with a tailored approach for each individual.

This study was approved by the Institutional Review Board of the Korea University Ansan Hospital (IRB No. AS2021-0278) and written informed consent was waived.

\section{CONFLICT OF INTEREST}

No potential conflict of interest relevant to this article was reported.

\section{ORCID}

Jinwook Hwang Jeong In Hong Hong Ju Shin Jae Seung Shin Je Hyeong Kim

https://orcid.org/0000-0003-4940-165X https://orcid.org/0000-0002-9660-7063 https://orcid.org/0000-0002-0731-3523 https://orcid.org/0000-0001-8147-6665 https://orcid.org/0000-0002-8995-7460

\section{AUTHOR CONTRIBUTIONS}

Conceptualization: JH. Data curation: JIH. Formal analysis: JH. Methodology: JHK, Project administration: JSS, HJS. Visualization: JH. Writing-original draft: JIH, JH. Writing-review\& editing: $\mathrm{JH}$. 

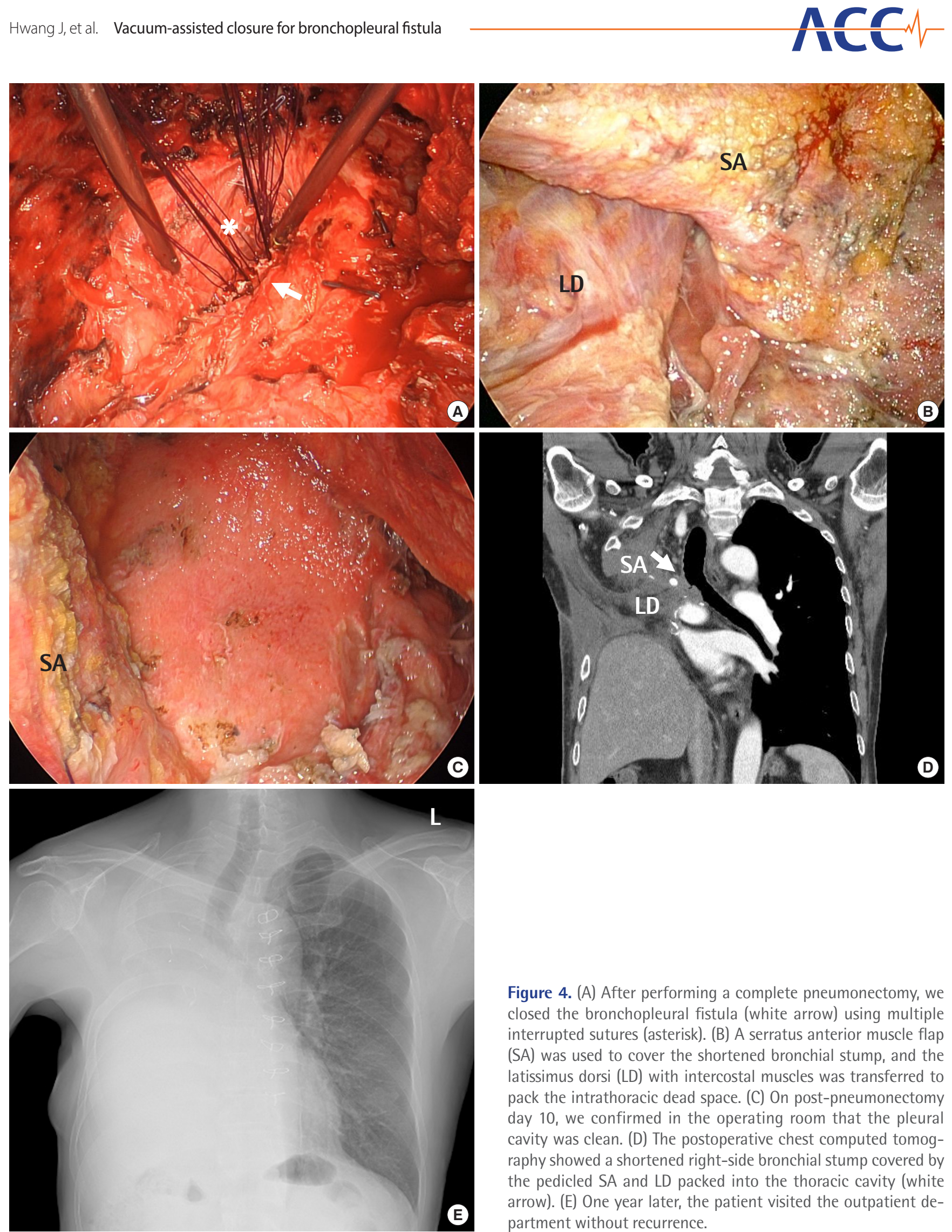

Figure 4. (A) After performing a complete pneumonectomy, we closed the bronchopleural fistula (white arrow) using multiple interrupted sutures (asterisk). (B) A serratus anterior muscle flap (SA) was used to cover the shortened bronchial stump, and the latissimus dorsi (LD) with intercostal muscles was transferred to pack the intrathoracic dead space. (C) On post-pneumonectomy day 10 , we confirmed in the operating room that the pleural cavity was clean. (D) The postoperative chest computed tomography showed a shortened right-side bronchial stump covered by the pedicled SA and LD packed into the thoracic cavity (white arrow). (E) One year later, the patient visited the outpatient department without recurrence. 


\section{REFERENCES}

1. Abruzzini P. Surgical treatment of fistulae of the main bronchus after pneumonectomy in tuberculosis (personal technic). Thoraxchirurgie 1963;10:259-64.

2. Fournier I, Krueger T, Wang Y, Meyer A, Ris HB, Gonzalez M. Tailored thoracomyoplasty as a valid treatment option for chronic postlobectomy empyema. Ann Thorac Surg 2012;94: 387-93.

3. Botianu PV, Botianu AM. Thoracomyoplasty in the treatment of empyema: current indications, basic principles, and re- sults. Pulm Med 2012;2012:418514.

4. Hysi I, Rousse N, Claret A, Bellier J, Pinçon C, Wallet F, et al. Open window thoracostomy and thoracoplasty to manage 90 postpneumonectomy empyemas. Ann Thorac Surg 2011;92: 1833-9.

5. Haghshenasskashani A, Rahnavardi M, Yan TD, McCaughan BC. Intrathoracic application of a vacuum-assisted closure device in managing pleural space infection after lung resection: is it an option? Interact Cardiovasc Thorac Surg 2011;13: 168-74. 